\title{
An Out-of-Plane Grating Coupler for Efficient Butt-Coupling Between Compact Planar Waveguides and Single-Mode Fibers
}

\author{
Dirk Taillaert, Member, IEEE, Wim Bogaerts, Member, IEEE, Peter Bienstman, Member, IEEE, \\ Thomas F. Krauss, Peter Van Daele, Ingrid Moerman, Member, IEEE, Steven Verstuyft, Kurt De Mesel, and \\ Roel Baets, Senior Member, IEEE
}

\begin{abstract}
We have designed and fabricated an out-of-plane coupler for butt-coupling from fiber to compact planar waveguides. The coupler is based on a short second-order grating or photonic crystal, etched in a waveguide with a low-index oxide cladding. The coupler is optimized using mode expansion-based simulations. Simulations using a 2-D model show that up to $74 \%$ coupling efficiency between single-mode fiber and a 240-nm-thick GaAs-AlO $x$ waveguide is possible. We have measured $19 \%$ coupling efficiency on test structures.
\end{abstract}

Index Terms-Integrated optics, optical planar waveguide components, waveguide coupler.

\section{INTRODUCTION}

$\mathbf{F}$ UTURE large-scale photonic integrated circuits will probably use photonic crystal waveguides or other compact planar waveguides. One of the major problems to be solved is the interface between the compact waveguide and the outside world. Coupling to a standard single-mode fiber using edge-coupling is a daunting task, because of the small dimensions of the waveguides (photonic crystal waveguides [1]-[3] are typically an order of magnitude smaller than conventional integrated optical waveguides). In particular, in high-vertical-index-contrast structures, such as silicon-on-insulator (SOI) or GaAs- $\mathrm{AlO}_{x}$, the light is strongly confined in a few hundred $\mathrm{nm}$ thin layer and coupling light into these waveguides is a problem. We propose the use of a grating coupler to butt-couple light from a single-mode fiber, perpendicular to the surface, into planar waveguides. This coupling scheme allows dense integration and wafer-scale testing because there is no need to cleave the devices. A sketch of the device is shown in Fig. 1. For this coupling scheme to work, two problems have to be solved. The light has to make a 90-degree turn

Manuscript received September 13, 2001; revised March 28, 2002. This work was supported in part by the European Union in the context of the IST project PICCO. The work of W. Bogaerts was supported in part by the Flemish Institute for the Industrial Advancement of Scientific and Technological Research (IWT) under a specialization grant. The work of P. Bienstman was supported by the Flemish National Fund for Scientific Research (FWO-Vlaanderen) under a doctoral fellowship.

D. Taillaert, W. Bogaerts, P. Bienstman, P. Van Daele, I. Moerman S. Verstuyft, K. De Mesel, and R. Baets are with the Department of Information Technology (INTEC), Ghent University-IMEC, 9000 Gent, Belgium (e-mail: Dirk.Taillaert@intec.rug.ac.be).

T. F. Krauss is with the School of Physics and Astronomy, St. Andrews University, St. Andrews KY16 9SS, U.K.

Publisher Item Identifier S 0018-9197(02)05710-X.

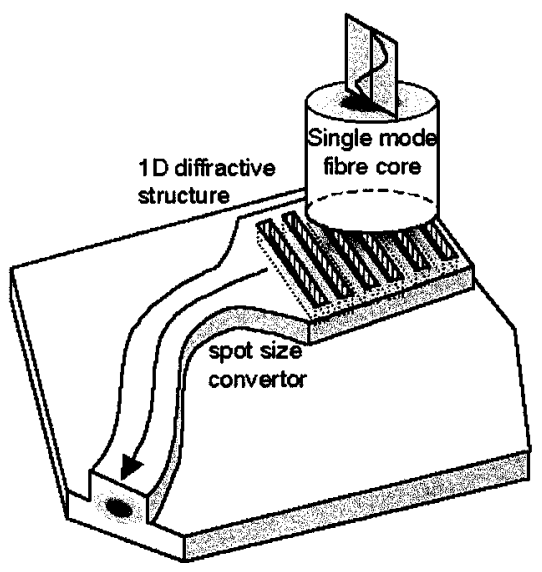

Fig. 1. Out-of-plane fiber coupler principle. The dimensions are not to scale for clarity. Only the core of the fiber is shown.

from the fiber to the waveguide and a broad (approximately $10 \mu \mathrm{m})$ waveguide has to be tapered into a narrow photonic crystal waveguide, preferably over a short distance. In the latter section, only a lateral spot-size convertor is needed, which is easier to achieve than a vertical spot-size convertor. In this paper we focus on the 90-degree bending problem.

Several grating couplers have been demonstrated that couple light out of [4]-[5] or into [6] waveguides. These couplers achieve high efficiencies $(>50 \%)$ but have a narrow bandwidth and they use relatively long $(>100 \mu \mathrm{m})$, weak gratings. In our design, the grating is much shorter $(10 \mu \mathrm{m}$ long) to be able to butt-couple to fiber. Therefore, the grating has to provide strong coupling and a relatively large overlap between the grating and the waveguide mode is needed. In a high-vertical-index-contrast structure, it is possible to achieve this with a shallow grating because of the strong vertical confinement.

\section{Computational Method AND StRucture Model}

A rigorous electromagnetic method is needed to design the coupler. Traditional grating theory which treats the grating as a small perturbation or of infinite extent is not accurate because our grating has only approximately 20 periods. A popular method to simulate photonic crystal structures of finite extent is the finite difference time-domain (FDTD) method [7]. Because we are using a waveguide structure and FDTD is rather slow, we prefer the eigenmode expansion technique 

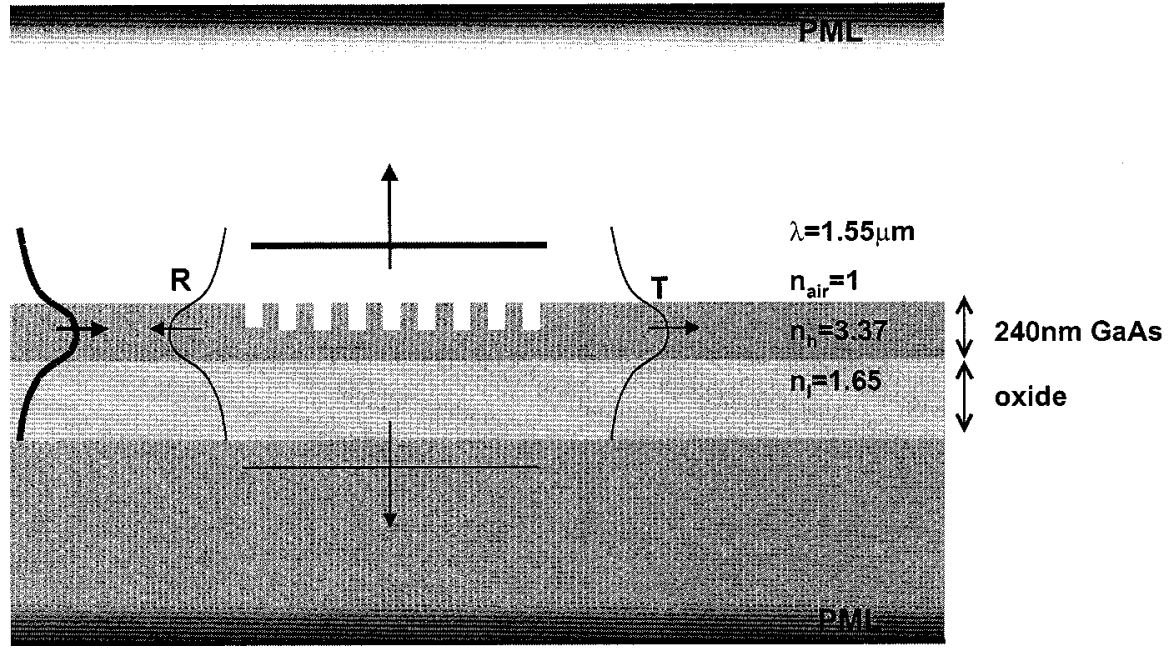

Fig. 2. Computational model used in simulations.

[8]. The boundary conditions are of utmost importance in our calculations because a lot of light is coupled out of a waveguide and heading straight for the boundaries of the computational domain. Perfectly matched layers (PMLs) [9] perform well and have also been introduced in mode expansion recently [10]. We used the CAMFR-simulation tool [11], which is based on eigenmode expansion and propagation with PML boundary conditions. In this work we consider only 1-D gratings in 2-D simulations and TE-polarization (E-field parallel to the grating grooves). This polarization also has a large bandgap for triangular lattices of air holes in a high index material [12] and is, therefore, interesting for photonic crystal based integrated circuits. It is expected that in a real 3-D configuration, the coupling efficiency will be lower than obtained with 2-D model-based simulations.

We calculate the coupling from waveguide to fiber, because this is easier with mode expansion. The coupling efficiency from fiber to waveguide is the same as from waveguide to fiber because we consider the coupling from one mode of a waveguide to one mode of another waveguide and the materials used are reciprocal. This powerful reciprocity principle is well known [13] and a mathematical proof based on Maxwell's equations is given in [14] for example. To avoid any doubt, we have also calculated the coupling from fiber to waveguide with 2-D FDTD simulations for the final designs and verified that it is the same as the calculated coupling from waveguide to fiber.

Our waveguide consists of a 240-nm-thick GaAs core with an oxide cladding on top of a GaAs substrate, as shown in Fig. 2. The thickness of the oxide will be optimized. A grating is etched into the GaAs layer. The top and bottom boundaries have PML to avoid parasitic reflections. The waveguide mode is incident from the left and is normalized so the input power equals 1 . In a first step, reflection and transmission of the entire structure are calculated. Afterwards, the field is calculated from which the power flux and overlap with a fiber mode can be calculated. The coupling efficiency to fiber is the fraction of the power in the waveguide mode that couples to the fiber. $R$ is the reflection at the waveguide grating interface, and $T$ is the transmission through the grating to the right.

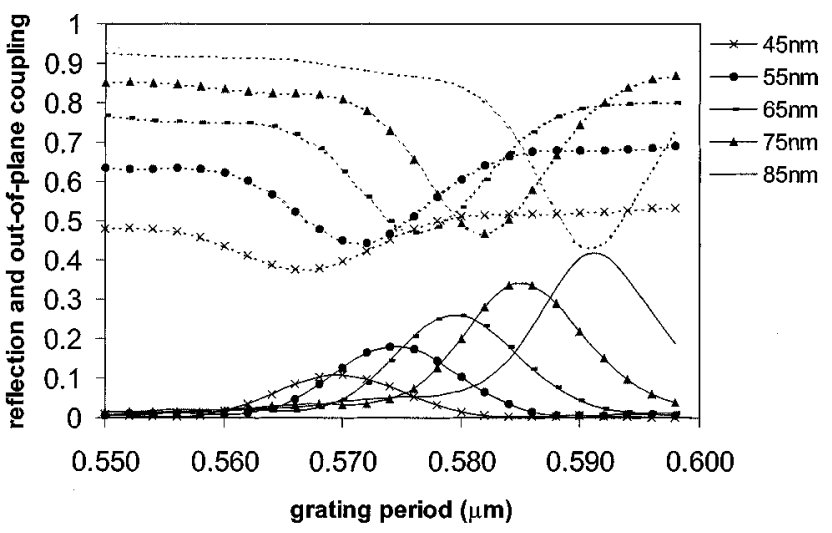

Fig. 3. Reflection (solid line) and out-of-plane coupling (dashed line) versus grating period for different etch depths. Out-of-plane coupling is defined as $1-R-T$ and consists of coupling to air/substrate and scattering at the interface. Grating duty cycle $=50 \%$.

\section{Simulation Results}

The coupler is based on a so-called second-order grating where the first-order diffraction couples light out of the waveguide, producing a surface-normal propagating field. An in-depth treatment of second-order gratings can be found in [15]. Because we use this grating as a coupler (first-order diffraction), we will use the term coupler grating instead of second-order grating in the rest of this paper. In this section, we describe the design and properties of the coupler grating and optimizations of the structure for optimal coupling efficiency. We start with a simple grating in part A and introduce enhancements in part B. All structures are designed for the 1550-nm wavelength.

\section{A. Simple Coupler Grating}

We can achieve 90-degree coupling with a grating when the grating period $\Lambda$ equals the wavelength divided by the effective refractive index $n_{\text {eff }}$. As the grating changes the effective index, the precise grating period has to be calculated numerically. Fig. 3 shows the reflection (solid lines) and out-of-plane coupling (dashed lines) as a function of $\Lambda$ for different etch 


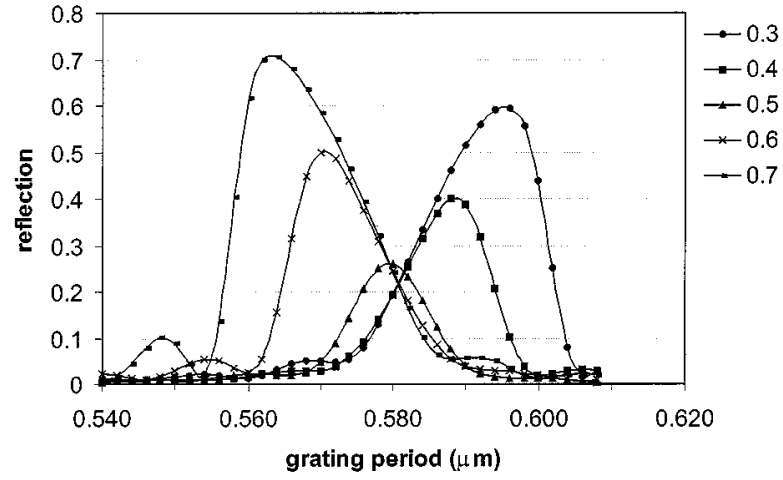

Fig. 4. Reflection versus grating period for different duty cycles. A 50\% duty cycle (triangles) has minimum reflection at resonance. Etch depth $=65 \mathrm{~nm}$.

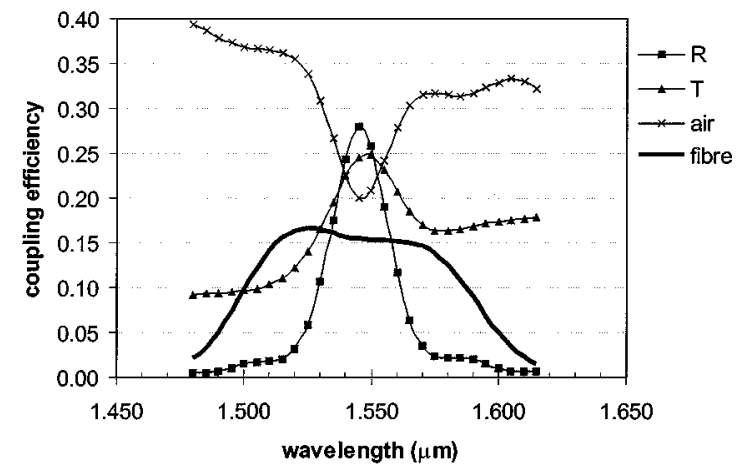

Fig. 5. Reflection (squares), transmission (triangles), top emitted power (crosses), and overall coupling efficiency to fiber (thick solid line) for 580-nm period, $65-\mathrm{nm}$ etch depth grating of 20 periods.

depths. Out-of-plane coupling is defined as $1-R-T$ and consists of the light that is coupled upwards and downwards and scattered at the interface. The reflection curves show a peak which corresponds to the resonance condition and 90-degree coupling. As the etch depth increases, $R$ increases and the resonant period shifts to larger periods (because $n_{\text {eff }}$ decreases). The out-of-plane coupling curves have a minimum at resonance. This minimum out-of-plane coupling has a maximum value for 65-nm etch depth. Previous results used a rectangular grating with 50\% duty cycle (equal tooth and groove widths). Reflection for different duty cycles is shown in Fig. 4. The duty cycle has a major impact on the amount of reflection. The resonance peak is smallest for the 50\% duty cycle. For $65-\mathrm{nm}$ etch depth and $50 \%$ duty cycle, the resonant grating period is $580 \mathrm{~nm}$. We will use these values as a starting point for our design.

For this grating $(\Lambda=580 \mathrm{~nm}$, etch depth $=65 \mathrm{~nm}$, duty cycle $=50 \%, 20$ periods), we have calculated the power that is coupled to the air and into the fiber in Fig. 5. To calculate the coupling efficiency to fiber, we use a Gaussian profile with a full width $(1 / e)$ of $10.5 \mu \mathrm{m}$ as a model of the fiber mode. Overall coupling efficiency to the fiber is $15 \%$ at $1550 \mathrm{~nm}$. Around the resonance wavelength, the outcoupling efficiency is rather low, but the coupling to the fiber is high. Away from the resonance wavelength, the outcoupling efficiency becomes higher but the coupling to the fiber is worse because of the phase mismatch. These two together cause a quite flat and large bandwidth. This wavelength dependence is only an approximation and will depend on the mode field diameter and NA of a particular fiber.

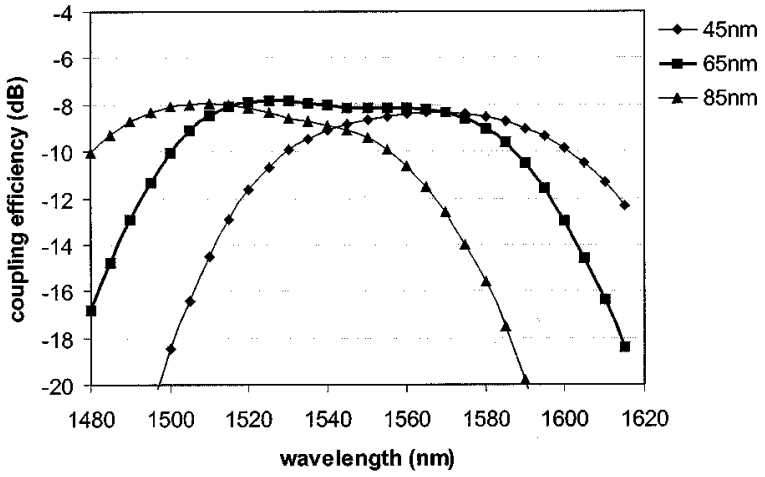

Fig. 6. Coupling efficiency and bandwidth (580-nm period, 50\% duty cycle) for different etch depths. Optimal etch depth $=65 \mathrm{~nm}$ (thick line).

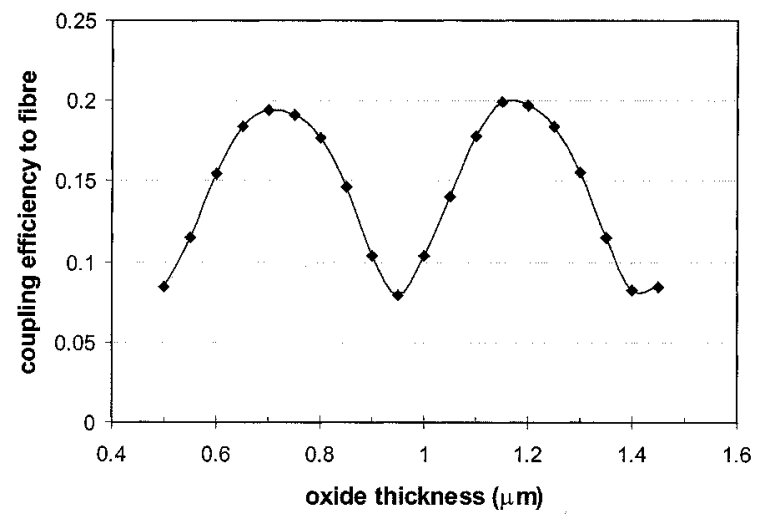

Fig. 7. Coupling efficiency versus oxide cladding thickness.

Fig. 6 shows the coupling efficiency versus wavelength for the same structure, but for different etch depths (fabrication tolerances). The 3-dB bandwidth is approximately $90 \mathrm{~nm}$ for the optimal etch depth ( $65 \mathrm{~nm}$, thick curve). These results were obtained for a structure with infinite oxide cladding thickness. We will now look at the more realistic structure with a finite oxide thickness and a high-index substrate. The oxide cladding thickness should be at least $0.5 \mu \mathrm{m}$, to avoid loss due to leakage of the guided mode to the substrate. The oxide thickness has little effect on the total amount of light that is coupled out, but has a serious impact on the air/substrate ratio [16]. A good choice of oxide thickness is important. From the coupling efficiency versus oxide thickness curve (Fig. 7), it can be seen that there is a 3-dB difference between the minima and maxima. The spacing between two maxima is half a wavelength, which confirms that this behavior is caused by interference between the direct upward wave and the reflection at the oxide/substrate interface.

The overall efficiency is $20 \%$ or $-7 \mathrm{~dB}$, for a 700 -nm-thick or 1200-nm-thick cladding. The efficiency is limited by reflection, transmission, and coupling to the substrate. To improve this value, reflection and transmission of the grating should be reduced and the directionality (air/substrate ratio) improved.

\section{B. Combined Coupler and Reflector Grating}

To reduce $R$ and $T$ at resonance, we propose a structure consisting of a grating coupler section and a first-order grating reflector [Fig. 8(a)]. It is possible to create a good reflector with a few periods when etching completely through the waveguide 
a)

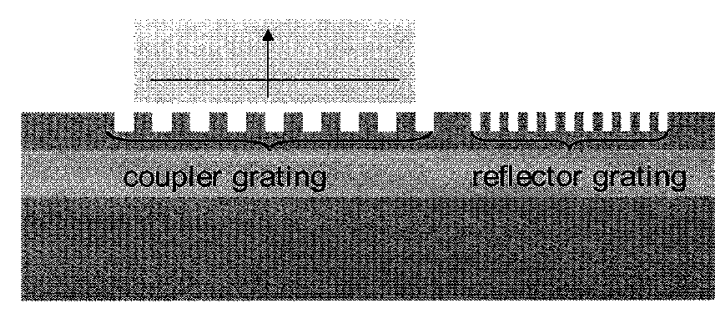

b)

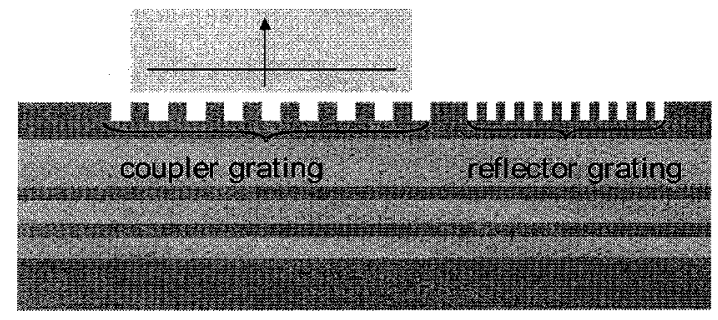

Fig. 8. (a) Combined coupler and reflector grating. (b) Same structure with a multilayer reflector under the waveguide.

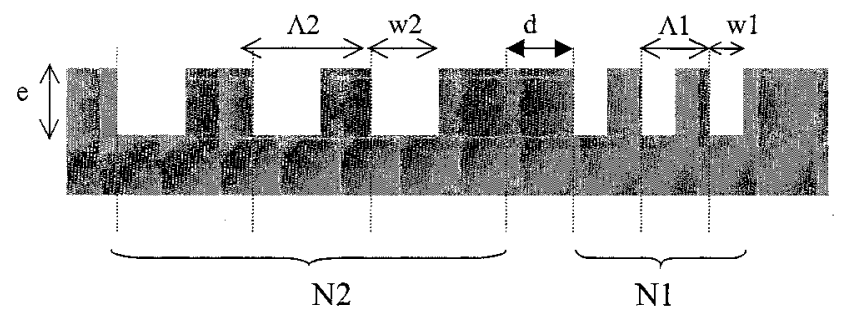

Fig. 9. Optimal grating parameters. The grating is etched in the GaAs waveguide core. Etch depth $e: 38 \mathrm{~nm}$. Period $\Lambda 2: 566 \mathrm{~nm}$. Groove width $w 2$ : $283 \mathrm{~nm}$. Number of periods $N 2: 20$. Spacing $d: 200 \mathrm{~nm}$. Period $\Lambda 1: 280 \mathrm{~nm}$. Groove width $w 1: 140 \mathrm{~nm}$. Number of periods $N 1: 70$.

(1-D photonic crystal). However, it is necessary to design a reflector grating with the same etch depth as the coupler grating because the spacing between the two gratings is critical and therefore they have to be fabricated in one step. We use the following procedure to optimize the lattice parameters. For a given etch depth, the coupler grating period is determined by the resonance condition as in part A. Afterwards, a first-order reflector grating is designed with the same etch depth. Finally, the spacing between the two gratings is optimized. This procedure is repeated for different etch depths and the final result has a maximum coupling efficiency to fiber of $38 \%$. The grating parameters are summarized in Fig. 9. With this structure, almost all light can be coupled out at the resonant wavelength, but the efficiency is still limited by coupling to the substrate. The maximum efficiency is $3 \mathrm{~dB}$ larger than the simple coupler grating, but the bandwidth is limited to $15 \mathrm{~nm}$, as can be seen in Fig. 10 . However, this bandwidth is quite large compared to the bandwidth of other grating couplers using long gratings.

To enhance the efficiency of grating couplers (avoid coupling to substrate), a blazed or parallellogram-shaped grating [17] is sometimes used. However, this makes simulation and fabrication much more difficult and the behavior of a blazed grating in our structure is yet unknown. Another solution to avoid coupling into the substrate is the addition of a distributed Bragg reflector (DBR) under the waveguide [Fig. 8(b)]. It is obvious that the spacing between the waveguide and the bottom reflector

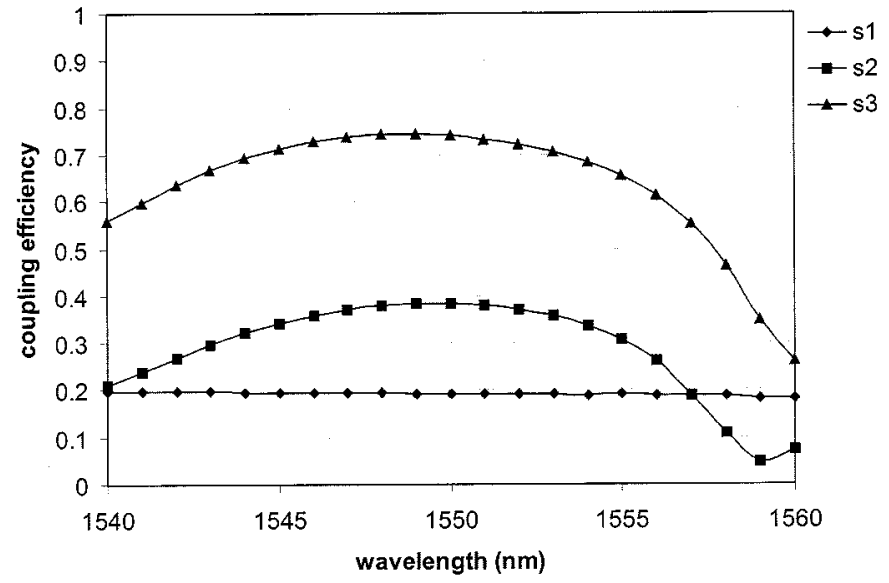

Fig. 10. Comparison of efficiency and bandwidth of the simple coupler grating (s1-diamonds), combined coupler and reflector grating (s2-squares), and the same structure with a multilayer bottom reflector ( $\mathrm{s} 3$ - triangles).

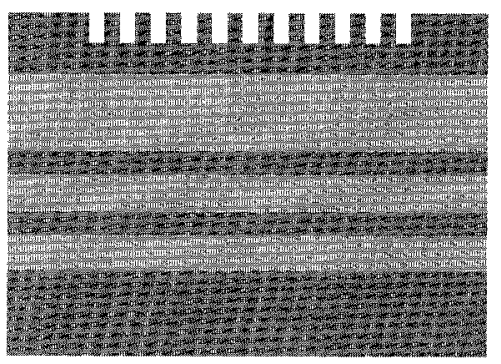

$240 \mathrm{~nm}$

$740 \mathrm{~nm}$

$115 \mathrm{~nm}$

$235 \mathrm{~nm}$

$115 \mathrm{~nm}$

235nm

Substrate

Fig. 11. Layer structure with a multilayer bottom reflector (dark grey is GaAs and light grey is $\mathrm{AlO}_{x}$ ).

will be important [15], but fabrication tolerances are reasonable because we are using a wavelength of $1550 \mathrm{~nm}$. Because of the large index contrast between GaAs and $\mathrm{AlO}_{x}$, only two mirror pairs are needed and therefore we will use the term multilayer reflector instead of DBR. In a first step, we design a multilayer reflector for maximum reflectivity. Afterwards, we optimize the thickness of the layer between the waveguide and multilayer reflector for maximum efficiency. The details of the layer structure can be found in Fig. 11, in which the grating is the same as in the structure without a multilayer reflector (Fig. 9). At the central wavelength, more than $95 \%$ of the light can be coupled upwards. Because of the coupling loss to the fiber, the overall efficiency is limited to $74 \%$. The bandwidth is similar to the structure without a multilayer reflector (Fig. 10). The most critical feature is the spacing between the coupler and the reflector grating. An error of a quarter wavelength will reduce the efficiency to almost zero (Fig. 12). Therefore, the fabrication of this structure requires state-of-the-art electron-beam lithography.

All our simulation results are for TE-polarization, and the coupling efficiency for TM is low (less than $-20 \mathrm{~dB}$ ) for all the structures described in this paper.

\section{PReliminary Experimental Results}

In this section, we describe our first experimental results. Devices were fabricated in GaAs-AlGaAs material grown by metal-organic vapor phase epitaxy (MOVPE) on a GaAs substrate (240-nm GaAs core/290-nm $\mathrm{Al}_{0.94} \mathrm{Ga}_{0.06} \mathrm{As}$ 


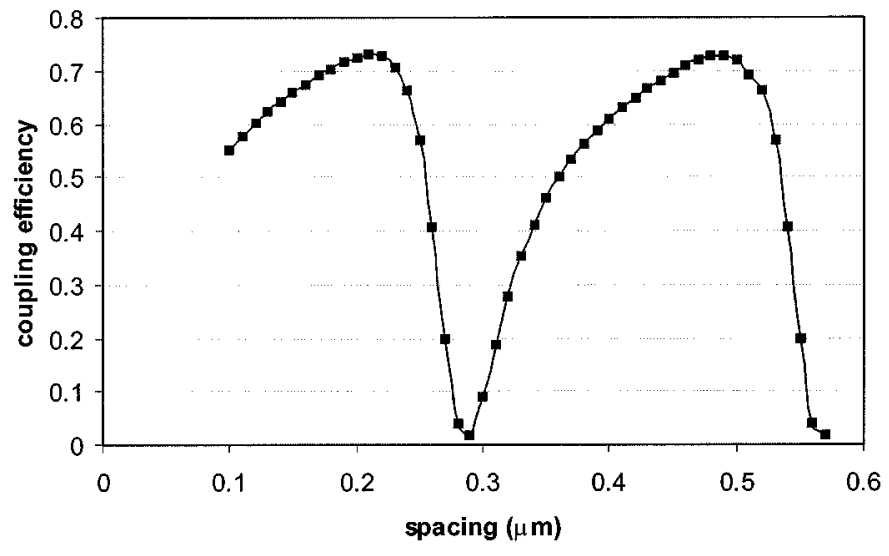

Fig. 12. Efficiency versus spacing between the coupler and reflector grating.

cladding/115 nm GaAs/240 $\mathrm{nm} \quad \mathrm{Al}_{0.94} \mathrm{Ga}_{0.06} \mathrm{As} / 115 \mathrm{~nm}$ $\left.\mathrm{GaAs} / 240 \mathrm{~nm} \mathrm{Al} \mathrm{l}_{0.94} \mathrm{Ga}_{0.06} \mathrm{As}\right)$. This layer structure has a multilayer reflector under the waveguide, but the thickness of the cladding layer differs from the optimized structure in Fig. 11. Because this difference is approximately half a wavelength, the calculated efficiencies are comparable.

The grating consists of a coupler and a reflector section (Fig. 9) and the calculated efficiency is $74 \%$. The gratings were fabricated using electron-beam lithography and reactive ion etching. Afterwards, ridge waveguides were defined using optical lithography and etching. The width of these waveguides is $10 \mu \mathrm{m}$ and the etch depth is $150 \mathrm{~nm}$. A last step was the etching of $20-\mu \mathrm{m}$-wide and $1500-\mathrm{nm}$-deep oxidation trenches and wet thermal oxidation of the $\mathrm{Al}_{0.94} \mathrm{Ga}_{0.06}$ As layers. The oxidized layers are mechanically stable and remain robustly attached to the GaAs layers.

For the measurements, we use a widely tunable laser source with 1-mW output power and polarization-maintaining output fiber (PMF). The TE-polarized light is coupled from the PMF via the grating into the waveguide. The output light from the waveguide facet is imaged onto a detector or an infrared (IR) camera. During initial alignment, we use the IR camera to monitor the waveguide spot. The output power versus wavelength for a $10-\mu \mathrm{m}$-wide ridge waveguide is shown in Fig. 13. A maximum efficiency of $14 \%(0.14 \mathrm{~mW}$ on detector) is measured in the 1515-1520-nm wavelength range. The shortest wavelength that is measured is $1510 \mathrm{~nm}$ because of the limitation on the tuning range of the laser. The 3-dB bandwidth is larger than 25 $\mathrm{nm}$. The fiber-to-waveguide distance is approximately $10 \mu \mathrm{m}$. We have measured the coupling efficiency from fiber to waveguide and not from waveguide to fiber because the latter requires coupling light into the waveguide at the edge, which is difficult.

The peaks in the measurements are similar to Fabry-Perot fringes and are caused by a cavity formed by the grating and a cleaved facet. The spacing between the peaks depends on the cavity length ( $300 \mu \mathrm{m}$ in this case). To estimate the actual coupling efficiency, we must take into account these cavity effects because the fiber-to-waveguide coupling efficiency is the power that would be measured when there is no reflection at the facet. When neglecting the waveguide propagation losses, the two parameters of the cavity are the facet reflection and the grating reflection. The facet reflection is 0.36 for our waveguide.

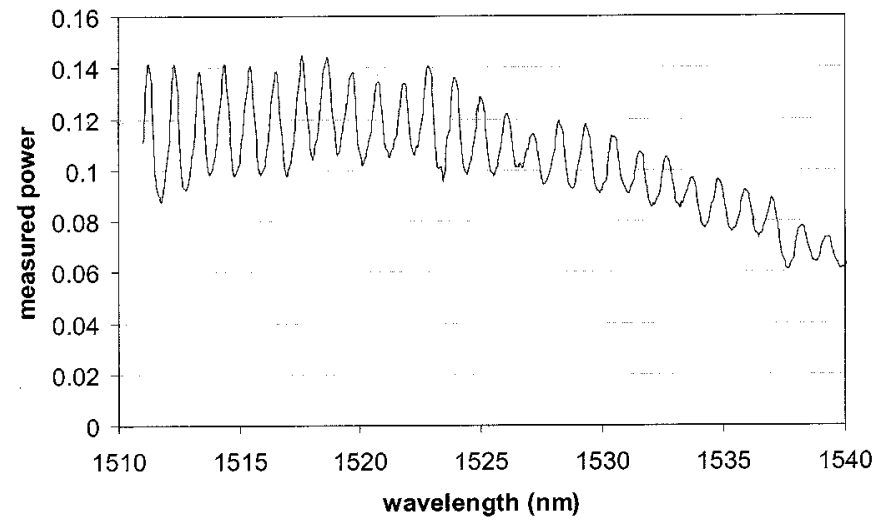

Fig. 13. Measured coupling efficiency versus wavelength. This is the optical power at the detector divided by the input power from the fiber.

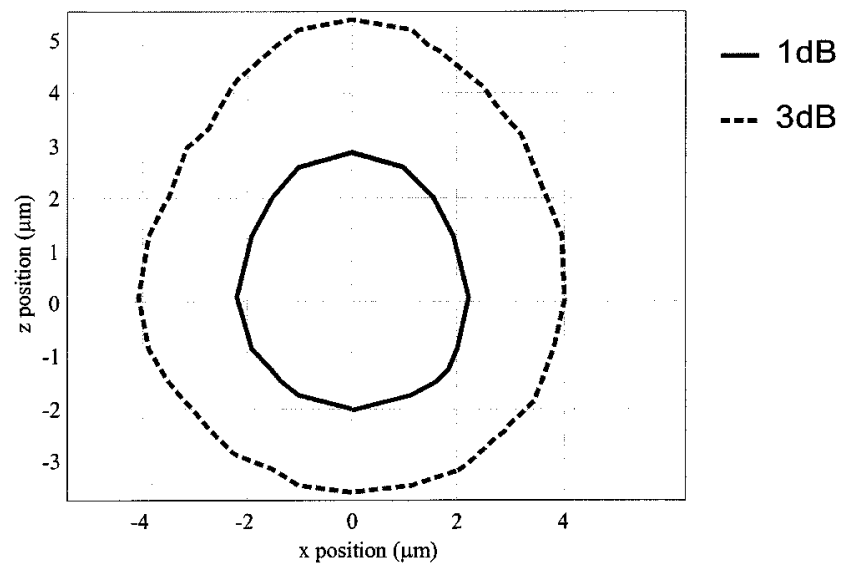

Fig. 14. Measured lateral alignment sensitivity. The $z$ axis is the waveguide propagation direction and the $x$ axis is parallel to the grating grooves; 1 - and 3-dB contour lines are shown.

The grating reflection is not known but can be estimated from $P_{\max } / P_{\min }$. Without any reflection at the grating, there is no cavity and $64 \%$ of the incoupled power reaches the detector. In our measurement results, $P_{\max } / P_{\min }$ is approximately 1.4 and the Fabry-Perot peaks correspond to $75 \%$ of the power that is coupled into the waveguide. Therefore, we estimate the actual coupling efficiency to be $19 \%(=0.14 / 0.75)$.

An advantage of the out-of-plane coupler compared to edge coupling is the relaxed lateral alignment sensitivity. The measured optical power as a function of the position of the fiber is shown in Fig. 14. A lateral deviation of $2 \mu \mathrm{m}$ in any direction results in less than $1 \mathrm{~dB}$ of additional coupling loss.

This preliminary experimental result shows that it is possible to obtain a reasonable efficiency with the out-of-plane fiber coupler. Further experimental work is in progress to improve the efficiency and achieve better agreement with the theoretical results.

\section{CONCLUSION}

We have presented the design of an out-of-plane coupler for coupling between single-mode fiber and compact planar waveguides. Using a 2-D model, we have shown coupling efficiencies of $38 \%$ for a $240-n m$-thick $\mathrm{GaAs}-\mathrm{AlO}_{x}$ waveguide or even $74 \%$ when adding a multilayer reflector under the waveguide. We 
have measured 19\% coupling efficiency on test structures. The proposed structure is a good candidate for solving the coupling problem to ultra-compact, high-vertical-index-contrast waveguides.

\section{ACKNOWLEDGMENT}

The authors gratefully acknowledge technical support by the Nanoelectronics Research Centre at Glasgow University.

\section{REFERENCES}

[1] M. Loncar, D. Nedeljcovic, T. Doll, J. Vuckovic, A. Scherer A, and T. P. Pearsall, "Waveguiding in planar photonic crystals," Appl. Phys. Lett., vol. 77, pp. 1937-1939, Sept. 2000.

[2] C. J. M. Smith, H. Benisty, S. Olivier, M. Rattier, C. Weisbuch C, and T. F. Krauss, "Low-loss channel waveguides with two-dimensional photonic crystal boundaries," Appl. Phys. Lett., vol. 77, pp. 2813-2815, Oct. 2000.

[3] A. Talneau, L. Le Gouezigou, and N. Bouadma, "1.55 $\mu \mathrm{m}$ low loss planar photonic crystal waveguides on InP substrate," in Proc. ECIO 2001, 2001, pp. 60-63.

[4] N. Eriksson, M. Hagberg, and A. Larsson, "Highly directional grating outcouplers with tailorable radiation characteristics," IEEE J. Quantum Electron., vol. 32, pp. 1038-1047, June 1996.

[5] A. Mekis, A. Dodabalapur, R. E. Slusher, and J. D. Joannopoulos, "Twodimensional photonic crystal couplers for unidirectional light output," Opt. Lett., vol. 25, pp. 942-944, July 2000.

[6] T. W. Ang, G. T. Reed, A. Vonsovici, A. G. R. Evans, P. R. Routley, and M. R. Josey, "Effects of grating heights on highly efficient unibond SOI waveguide grating couplers," IEEE Photon. Technol. Lett., vol. 12, pp. 59-61, Jan. 2000.

[7] A. Taflove, Computational Electrodynamics, the Finite-Difference TimeDomain Method. Norwood, MA: Artech House, 1995.

[8] G. Sztefka and N. Holting, "Bidirectional eigenmode propagation for large refractive index steps," IEEE Photon. Technol. Lett., vol. 5, pp. 554-557, 1993.

[9] J. P. Berenger, "A perfectly matched layer for the absorption of electromagnetic waves," J. Comput. Phys., vol. 114, pp. 185-200, Oct. 1994.

[10] P. Bienstman, H. Derudder, R. Baets, F. Olyslager, and D. De Zutter, "Analysis of cylindrical waveguide discontinuities using vectorial eigenmodes and perfectly matched layers," IEEE Trans. Microwave Theory Tech., vol. 49, pp. 349-354, Feb. 2001.

[11] P. Bienstman and R. Baets, "Optical modeling of photonic crystals and VCSEL's using eigenmode expansion and perfectly matched layers," Opt. Quantum Electron., vol. 33, pp. 327-341, Apr. 2001.

[12] J. D. Joannopoulos, R. D. Meade, and J. N. Winn, Photonic Crystals: Molding the Flow of Light. Princeton, NJ: Princeton Univ. Press, 1995.

[13] R. Harrington, Time-Harmonic Electromagnetic Fields. New York: McGraw-Hill, 1961.

[14] F. Olyslager, Electromagnetic Waveguides and Transmission Lines. Oxford, U.K.: Oxford Univ. Press, 1999, pp. 79-83.

[15] A. Hardy, D. F. Welch, and W. Streifer, "Analysis of 2nd-order gratings," IEEE J. Quantum Electron., vol. 25, pp. 2096-2105, Oct. 1989.

[16] T. Suhara and H. Nishihara, "Integrated optics components and devices using periodic structures," IEEE J. Quantum Electron., vol. 22, pp. 845-867, June 1986

[17] M. Hagberg, N. Eriksson, and A. Larsson, "High efficiency surface emitting lasers using blazed grating outcouplers," Appl. Phys. Lett., vol. 67, pp. 3685-3687, Dec. 1995.

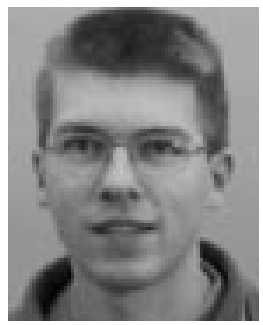

Dirk Taillaert (M'97) received the degree in electrical engineering from the University of Gent, Belgium, in 1999, where he is currently working toward the $\mathrm{Ph} . \mathrm{D}$. degree in electrical engineering in the Department of Information Technology.

His research interests include design, fabrication, and characterization of waveguides and components for microphotonics. He is currently working on waveguide-fiber interfaces.

Mr. Taillaert is a member of the IEEE Lasers and Electro-Optics Society.

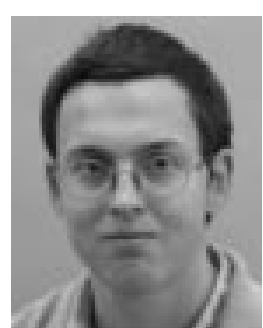

Wim Bogaerts (M'97) received the degree in engineering (physics) from Gent University, Gent, Belgium, in 1998, where he is currently working toward the $\mathrm{Ph} . \mathrm{D}$. degree in electrical engineering in the Department of Information Technology.

His research interests include integrated optics in general and on design, application, and fabrication of photonic crystals in particular.

Mr. Bogaerts is a member of the IEEE Lasers and Electro-Optics Society.

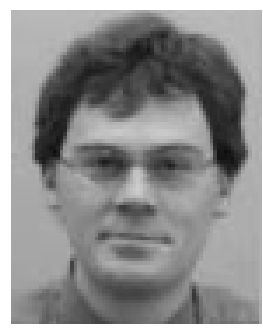

Peter Bienstman (M'96) was born in Gent, Belgium, in 1974. He received the degree in electrical engineering and the Ph.D. degree from Ghent University, Gent, Belgium, in 1997 and 2001, respectively.

$\mathrm{He}$ is currently spending a year as a Post-Doctoral Researcher at the Massachusetts Institute of Technology, Cambridge. His research interests include the modeling of optical structures, notably photonic crystal structures, vertical-cavity surface-emitting lasers, and resonant-cavity light-emitting diodes. He has published several papers and has one patent

application pending.

Dr. Bienstman is a member of the IEEE Lasers and Electro-Optics Society.

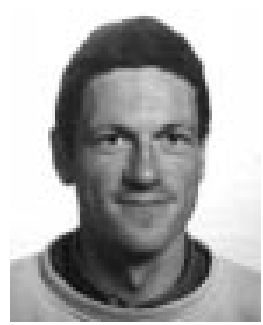

Thomas F. Krauss received the Ph.D. degree from the University of Glasgow, U.K., where his work focused on semiconductor ring lasers.

$\mathrm{He}$ has been involved in integrated optics research since 1989. While at the University of Glasgow, he demonstrated low threshold current levels and greatly improved external device efficiency. In 1993, he began work on photonic crystals, via a series of fellowships (EPSRC 1993; Royal Society 1995-2003) and became one of the pioneers of semiconductor-based photonic crystals. In 1997, he spent one year at the California Institute of Technology, Pasadena, working on efficient photonic crystal based light emitters. In 2000, he moved to a personal chair in Optoelectronics at the School of Physics and Astronomy, University of St. Andrews, U.K., and set up a photonic crystal research group (currently ten members) and a semiconductor microfabrication facility. He is grant holder of several U.K. research projects and is coordinator of the European research project "PICCO."

Dr. Krauss was Organizer and Chairman of the trend-setting Workshop "PECS3" in St. Andrews in June 2001 and is on the committee of several other workshops and summer schools, including MRS and PECS. He is a fellow of the Royal Society of Edinburgh.

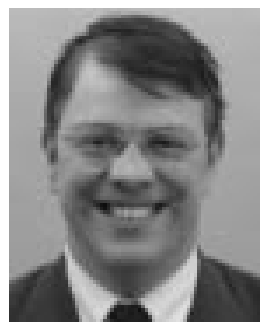

Peter Van Daele received the Ph.D. degree in electrical engineering from the University of Ghent, Gent, Belgium, in 1998

He became a permanent member of staff of the Interuniversity Micro-Electronics centre (IMEC) of Department of Information Technology of the University of Ghent, where he was responsible for the research on processing of III-V optoelectronic devices. Since 2001, his work has more focused on optical packaging and optical interconnections, with emphasis on coupling to fiber arrays, integration on printed circuit boards and use of laser processing techniques. In 1993, he also became a part-time professor. He is author or co-author of about 200 publications in the field of optoelectronic components and technology. 


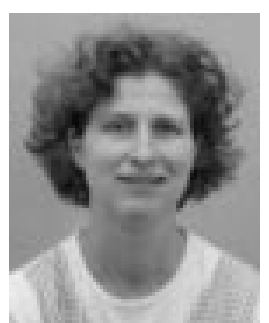

Ingrid Moerman (M'96) received the degree in electrical engineering and the Ph.D. degree from Ghent University, Gent, Belgium, in 1987 and 1992, respectively.

Since 1987, she has been with the Interuniversity Micro-Electronics centre (IMEC) at the Department of Information Technology (INTEC) of the Ghent University. Her main contributions are the epitaxial growth of III/V opto-electronic components and photonic integrated circuits for telecom applications. In 1997, she became a permanent member of the Research Staff at IMEC, where she coordinates the research on the epitaxial growth of III-V opto-electronic devices. Since 2000, she is part-time professor at Ghent University. She has recently expanded her research domain to broadband communication networks. She is author or co-author of more than 250 publications in the field of opto-electronic components and technology.

Dr. Moerman is a member of the IEEE Lasers and Electro-Optics Society and the IEEE Communications Society.

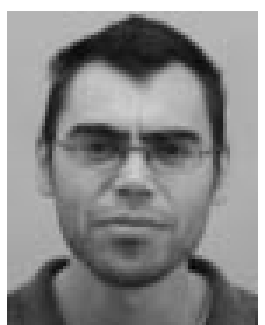

Steven Verstuyft is responsible for the processing of III-V optoelectronic devices at the Department of Information Technology of the University of Ghent, Gent, Belgium.

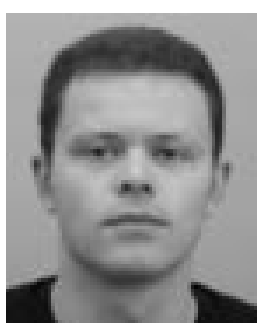

Kurt De Mesel was born in Poperinge, Belgium, in 1972. He received the degree in electrical engineering from the University of Ghent, Gent, Belgium, in 1997, where he is currently working toward the Ph.D. degree in electrical engineering.

His research interests include modeling, design and fabrication of spot-size converters and oxide-confined devices.

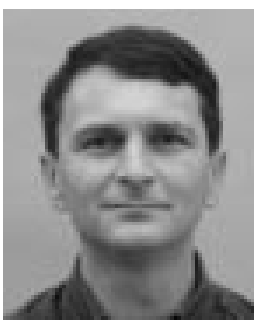

Roel Baets (M'88-SM'96) received the degree in electrical engineering from Ghent University, Gent, Belgium, in 1980, the M.Sc. degree in electrical engineering from Stanford University, Stanford, CA, in 1981, and the Ph.D. degree from Ghent University in 1984.

Since 1981, he has been with the Department of Information Technology (INTEC) of Ghent University. Since 1989, he has been a Professor on the Engineering Faculty of Ghent University. From 1990 to 1994, he was also a part-time professor at the Technical University of Delft, The Netherlands. He has mainly worked in the field of III-V devices for optoelectronic systems. With about 300 publications and conference papers as well as about ten patents, he has made contributions to the design and fabrication of semiconductor laser diodes, passive guided wave devices, PICs, and microoptic components. He leads the Optoelectronic Components and Systems group at Ghent University-INTEC (which is an associated lab of IMEC), working on photonic devices for optical communication and optical interconnect.

Dr. Baets was Chairman of the IEEE LEOS Benelux Chapter from 1999 to 2001. He is a member of the Optical Society of America, the IEEE Lasers and Electro-Optics Society, SPIE, and the Flemish Engineers Association. He has been a member of the program committees of OFC, ECOC, IEEE Semiconductor Laser Conference, ESSDERC, CLEO-Europe, and the European Conference on Integrated Optics. 